\title{
A formal and integrated framework to simulate evolution of biological pathways
}

\author{
Lorenzo Dematté \\ The Microsoft Research - University of Trento \\ Centre for Computational and Systems Biology \\ dematte@cosbi.eu \\ Corrado Priami \\ The Microsoft Research - University of Trento \\ Centre for Computational and Systems Biology \\ priami@cosbi.eu \\ Alessandro Romanel \\ The Microsoft Research - University of Trento \\ Centre for Computational and Systems Biology \\ romanel@cosbi.eu
}

Orkun S. Soyer

The Microsoft Research - University of Trento Centre for Computational and Systems Biology

soyer@cosbi.eu

This is the preliminary version of a paper that will appear in Theoretical Computer Science

available at http://www.elsevier.com/wps/find/journaldescription.cws_home/ 505625/description\#description 


\begin{abstract}
We present a formal approach to the study of evolution of biological pathways. The basic idea is to use the Beta Workbench to model and simulate pathway in connection with evolutionary algorithms to implement mutations. A fitness function is used to select individuals at any generation. The feasibility of the approach is demonstrated on the MAPK pathway.
\end{abstract}

\title{
1 Introduction
}

Biological pathways are studied using several methods, including but not limited to analysis of their single components, reconstruction of their series of chemical reactions and simulation of their dynamics.

Many approaches include modelling techniques to represent and analyse dynamics of complex pathways. In particular, process calculi have been recently applied to model and simulate biological systems [1]. The main activities up to now have been oriented to define new primitives and to show their feasibility for describing biological phenomena. Some simulation engines have been developed especially based on the stochastic $\pi$-calculus [2] like SPiM [3] or BIOSPI [4], and recently on an extension of the Beta-binders language $[5]^{1}$.

Recently, there is an interest in using evolutionary approaches to study pathways. Understanding how pathways emerged during evolution can help us to understand their basic properties, such as the role of complexity and the importance of topology and feedback loops. Existing approaches to study evolution are commonly based on comparative genomics or proteomics and on phylogenetic analysis $[6,7]$. These studies compare pathways from different organisms to see how evolution affects the internal structure of the network of interactions.

An alternative approach is to simulate evolution in silico, using an algorithm that mimic this process, as in $[8,9,10,11]$. However, these approaches use ad-hoc tools and representations of pathway dynamics, while current available tools to model and simulate pathway dynamics, discussed above, do not allow for evolutionary simulations.

On the other hand, tools that mimic evolution are also known in the Computer Science domain. However, these tools and algorithms are designed for specific tasks, such as machine learning and optimization or search problems [12], and therefore they lost their strict connection with biology and they are no more adequate to study biological evolution in a realistic way.

Here, we aim at developing a specific framework to allow straightforward study of pathway evolution based on the Beta Workbench (BetaWB) [5].

\footnotetext{
${ }^{1}$ Available at url http://www.cosbi.eu/Rpty_Soft_BetaWB.php
} 
The great flexibility of the Beta-binders process calculus in the definition of the structure of proteins allows us to introduce interesting primitives for mutations used to build domain-based interaction and mutation models. Moreover, pathway dynamics can be easily modelled, and the interactions of emerged pathways analysed. Our formal framework aims at extending the features of the BetaWB to obtain a full integration with the existing software environment. In this paper we describe the novelty of the approach unravelling the formal theory on which it is based, and we show the feasibility of the proposed solution to study evolution of pathways.

The paper is organised as follows. In the next section we briefly recall the language and the software tool BetaWB on top of which we built our work for simulating the evolution of biological pathways. The model we used to describe the general structure of signalling pathways is also introduced and explained. Sect. 3 discusses the evolutionary framework, the integration of the BetaWB simulation engine within an evolutionary algorithm and how mutations are performed on the model. An example of application of the whole framework is given in Sect. 4, and conclusions and future work directions close the paper.

\section{Description of pathway dynamics}

To study biological evolution of pathways, we need to describe pathway dynamics and an algorithm to simulate pathway evolution from a generation to the next one. We start describing the modeling and simulation tool.

\subsection{The Beta Workbench}

The Beta Workbench is a software framework for modelling and simulating biological processes [5]. It incorporates a language, a compiler to a stochastic abstract machine, the execution environment and some graphical interface components. The BetaWB language is based on $\beta$-binders [13], a process calculus developed for better representing the interactions between biological entities.

\subsubsection{The Language}

A BetaWB program, called also $\beta$-system, is a tuple $Z=\langle B, E, \xi\rangle$ made up of a bio-process $B$, a list of events $E$ and ambient $\xi$. The bio-process $B$ intuitively represents the structure of the system, that is a set of entities interacting in the same environment, $E$ represents the list of possible events enabled on the system and the ambient $\xi$ contains information about the environment, like the set $T$ of the considered types (ranged over by $\Delta, \Gamma_{0}$, 
$\left.\Sigma^{\prime}, \cdots\right)$, a function $\rho: \mathcal{N} \rightarrow \mathbb{R}$ that associates stochastic rates ${ }^{2}$ to names in $\mathcal{N}$ and the function $\alpha: T^{2} \rightarrow \mathbb{R}^{3}$, which describes the affinity relation between couples of types. In particular, given two types $\Delta$ and $\Gamma$, the application of $\alpha(\Delta, \Gamma)$ returns a triple of stochastic rates $(r, s, t)$, where the value $r$, denoted with $\alpha_{c}(\Delta, \Gamma)$, represents the complexation rate, the value $s$, denoted with $\alpha_{d}(\Delta, \Gamma)$, represents decomplexation rate and the value $t$, denoted with $\alpha_{i}(\Delta, \Gamma)$, represents the inter-communication rate of the two entities exposing the types $\Delta$ and $\Delta$.

The syntax of the BetaWB language is the following.

$$
\begin{aligned}
& B \quad::=\operatorname{Nil}|\vec{B}[P]| B \| B \\
& \vec{B} \quad::=\widehat{\beta}(x, r, \Delta) \mid \widehat{\beta}(x, r, \Delta) \vec{B} \\
& \widehat{\beta} \quad::=\beta\left|\beta^{h}\right| \beta^{c} \\
& P \quad::=\text { nil }|P| P|! \pi . P| M \\
& M \quad::=\pi \cdot P \mid M+M \\
& \pi \quad::=x(y)|\bar{x}\langle y\rangle|(\tau, r) \mid(\text { die }, r)|(\operatorname{ch}(x, \Delta), r)| \\
& \text { (hide }(x), r) \mid \text { (unhide }(x), r) \mid(\operatorname{expose}(x, s, \Delta), r) \\
& \text { cond } \quad::=\vec{B}[P]: r|| \vec{B}[P]|=n| \vec{B}[P], \vec{B}[P]: r \\
& \text { verb }::=\operatorname{new}(\vec{B}[P], n)|\operatorname{split}(\vec{B}[P], \vec{B}[P])| \operatorname{join}(\vec{B}[P]) \mid \text { delete } \\
& \text { event }::=\bullet \mid(\text { cond }) \text { verb } \\
& E \quad::=\text { event } \mid \text { event }:: E
\end{aligned}
$$

where $x, y \in \mathcal{N}, n \in \mathbb{N}$ and $r \in \mathbb{R}^{+} \cup\{\infty\}$ is a stochastic rate.

Processes generated by the non terminal symbol $P$ are referred as $p i$ processes. Bio-processes(boxes) are defined as pi-processes prefixed by specialised binders $\vec{B}$ that represent interaction capabilities. An elementary beta binder $\widehat{\beta}$ has the form $\beta(x, r, \Gamma)$ (active), $\beta^{h}(x, r, \Gamma)$ (hidden) or $\beta^{c}(x, r, \Gamma)$ (complexed), where the name $x$ is the subject of the beta binder and $\Gamma$ represents the type of $x$. Although types can be any general structure for which exist a decidable equality relation, without loss of generality we assume here that types are names taken from a countably infinit set $T$ such that $T \cap \mathcal{N}=\emptyset$. A bio-process $B$ is either the deadlock beta-process $N i l$ or a parallel composition of boxes $\vec{B}[P]$. A box $\vec{B}[P]$ models a single biological entity, where intuitively the beta binders list $\vec{B}$ represents the interface of the entity, while the pi-process $P$ represents its internal structure or processunit. Moreover, the language is provided with a graphical representation of boxes:

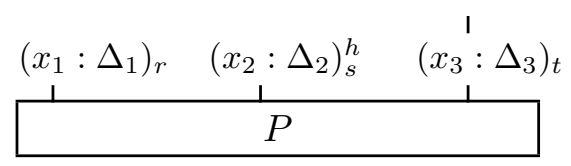

\footnotetext{
${ }^{2} \mathrm{~A}$ stochastic rate is the single parameter defining an exponential distribution that drives the stochastic behaviour of an action. The rate $\infty$ is used to denote immediate actions, i.e., actions that are executed as soon as they become enabled.
} 
The pairs $x_{i}: \Delta_{i}$ represent the sites through which the box may interact with other boxes. Types $\Delta_{i}$ express the interaction capabilities at $x_{i}$. The value $r$ represents the stochastic rate associated to the name $x$ inside the box, $h$ represents the hidden status and the black line over the last beta binder represents the complexed status.

The dynamics of the system is formally specified through the operational semantics of the language available in [5], which uses a notion of structural congruence $\equiv$. Intuitively, that two $\beta$-systems $Z=\langle B, E, \xi\rangle$ and $Z^{\prime}=\left\langle B^{\prime}, E^{\prime}, \xi^{\prime}\right\rangle$ are structurally congruent $\left(Z \equiv Z^{\prime}\right)$, if their bio-processes $B$ and $B^{\prime}$ and their list of events $E$ and $E^{\prime}$ are identical up to structure $\left(B \equiv_{b} B^{\prime}\right.$ and $\left.E \equiv_{e} E^{\prime}\right)$ and their ambients are equal $\left(\xi=\xi^{\prime}\right)$. Moreover, two boxes representing interacting entities, are considered of the same species only if they are structurally congruent. Given a $\beta$-system, its temporal evolution is described by three types of actions.

Monomolecular actions describe the dynamics of single boxes. More precisely, an intra-boxes communication allows components to interact within the same box, the expose action adds a new site of interaction to the interface of the box containing the expose, the change action modifies the type of an interaction site, hide and unhide actions make respectively invisible and visible an interaction site. Finally, the die action eliminates the box that performs the action and, recursively, all the boxes directly or indirectly complexed with them.

Bimolecular actions describe interactions that involves two boxes. The complex operation creates a dedicated communication binding between boxes over compatible and unhidden elementary beta binders, while the decomplex operation destroys an already existing dedicated binding. The stochastic rates associated to complex and decomplex operations are, respectively, the complexation and decomplexation rates derived from the affinity function. In the example, the complexation rate is $\alpha_{c}(\Delta, \Gamma)$, while the decomplexation rate is $\alpha_{d}(\Delta, \Gamma)$. The information about the existing dedicated bindings is maintained in the environment. The last bimolecular action is the inter-boxes communication, which enables interaction between boxes over compatible and unhidden elementary beta binders. Suppose $\Delta$ and $\Gamma$ be the types associated to the involved elementary beta binders. If $\alpha_{c}(\Delta, \Gamma)>0$, then the inter-communication is enabled, with rate $\alpha_{i}(\Delta, \Gamma)$, only after a dedicated communication binding, over the involved beta binders, has been created by a complex operation. Otherwise, the inter-boxes communication is simply enabled with rate $\alpha_{i}(\Delta, \Gamma)$.

An Event is the composition of a condition cond and an action verb and it is triggered only when the condition associated with the event is satisfied. Events can be considered as global rules of the system which can substitute, create and delete boxes from the system. In particular, the list $E$ can contain five types of events: join, which substitutes two boxes with single ones; split, which substitutes a box with two boxes; new, which introduces a specified 
number of instances of a box; delete, which eliminates boxes.

Definition 1 The BetaWB Stochastic Transition System (STS) is referred as $\mathcal{S}=\left(\mathcal{Z}, \stackrel{r}{\rightarrow}_{s}, Z_{0}\right)$, where $\mathcal{Z}$ is the set of $\beta$-systems, $Z_{0} \in \mathcal{Z}$ is the initial $\beta$-system and $\stackrel{r}{\rightarrow}_{s} \subseteq \mathcal{Z} \times \mathbb{R} \times \mathcal{Z}$ is the stochastic transition relation, where $r$ is a stochastic rate constant and is derived using information in the syntax and in the ambient of the $\beta$-system.

For a more detailed and formal description of the language, its operational semantics and the laws of its structural congruence relation we refer the reader to [5].

\subsubsection{Simulator and Companion Tools}

The BetaWB simulator is the core part of the Beta Workbench and it is built as a composition of three logical blocks: the compiler, the runtime environment and the stochastic simulation engine.

The BetaWB simulator is a command-line application that takes as input two text files that describe an initial $\beta$-system $Z=\langle B, E, \xi\rangle$. The first file describes the bio-process $B$ and the list of events $E$ with a simple functionallike syntax, while the second one contains the specification of the set of types $T$, the affinity function $\alpha$ and the function $\rho$.

The compiler translates these files into a runtime representation that is then stored into the runtime environment. The runtime environment provides the stochastic simulation engine with primitives for checking the current state of the system and for modifying it. The stochastic simulation engine handles the time evolution of the environment in a stochastic way and preserves the semantics of the language. The stochastic simulation engine implements an efficient variant of the Gillespie's algorithm described in [14].

The BetaWB designer is a graphical tool that allows the user to write $\beta$ system in a graphical way (Fig.1). In particular, it is possible to draw boxes, pi-processes, interactions, events and to form complexes using graphs. The graphical format and the textual description of the $\beta$-system are interchangeable: the tool can parse and generate the graphical representation from any valid $\beta$-system, and generate the textual representation from the graphical form. The textual representation can then be used as input of the BetaWB simulator.

The BetaWB plotter is a graphical tool that parses and display simulation outputs.

\subsection{A compositional model for signalling pathways}

A signalling pathway is any biological process that converts one kind of signal or stimulus into another; this conversion is also called signal transduction. In general, a signalling pathway results in a composition or cascade 


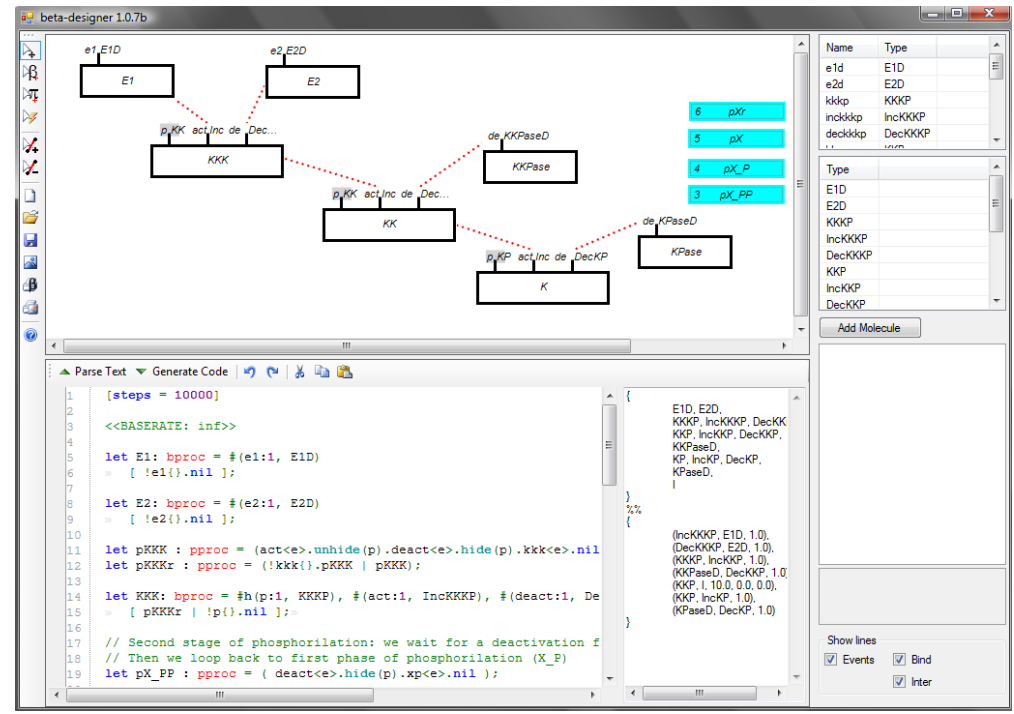

Figure 1: BetaWB designer: example of graphical representation.

of biochemical reactions that are carried out by proteins and linked through second messengers. Biological signal transduction allows a cell or organism to sense its environment and react accordingly. Typically, a signalling pathway has one (or more) input, represented by any environmental stimulus, and one (or more) output, represented by an active protein.

In the BetaWB language we represent a protein as a biological entity composed by a set of sensing domains, a set of effecting domains and an internal structure. Sensing domains are the places where the protein receives signals, effecting domains are the places that a protein uses for propagating signals, and the internal structure codifies for the mechanism that transforms an input signal into a protein conformational change, which can result in the activation or deactivation of another domain. This is inspired by the available knowledge of protein structure and function (see for example [15]).

As explained in Sec.2.1, the Beta Workbench is a general framework for modeling and simulating biological processes; each biological entity is modelled with a box, which is a composition of an interface and an internal process unit. This gives an effective way for modeling proteins by decomposing the domains of interaction and the internal structure into two different constructs. Moreover, the compositional nature of the language allows us to design and apply mutations on biological entities in an effective, simple and intuitive way.

In this application of evolutionary algorithms to $\beta$-systems we designed a general methodology for modeling proteins by providing patterns for modeling interaction domains and internal structures. Domains are represented using beta binders ( $A+$ and $A-$ ), interaction sites with an affinity. As shown 
in Fig. 2 (where we omit subjects and rates of beta binders for the sake of readability and where arrows indicate that types associated to binders are compatible), the sensing domain is represented by a pair of binders, one for receiving a signal of activation (e.g. phosphorylation) and the other for receiving a signal of deactivation (e.g. dephosphorylation) sent to the protein. The effecting domain $(A o)$ is instead used to communicate, and so to activate or inhibit, other proteins.

Pattern of pi-processes and boxes for modeling proteins with single sensing and effecting domains are the following:

$$
\begin{aligned}
& p s_{1} \triangleq \overline{a c t}\langle\epsilon\rangle \cdot(\text { unhide }(o), \infty) \cdot \overline{\operatorname{deact}}\langle\epsilon\rangle \cdot(\operatorname{hide}(o), \infty) \cdot \bar{x}\langle\epsilon\rangle \cdot \text { nil } \\
& p r_{1} \triangleq ! x() \cdot p s_{1} \mid p s_{1} \\
& p s s_{2} \triangleq \overline{\operatorname{deact}}\langle\epsilon\rangle \cdot(\operatorname{hide}(o), \infty) \cdot \overline{x p}\langle\epsilon\rangle \cdot \text { nil } \\
& p s_{2} \triangleq \operatorname{lxp}() \cdot\left(\overline{a c t}\langle\epsilon\rangle \cdot(\text { unhide }(o), \infty) \cdot p s s_{2}+\overline{\operatorname{deact}}\langle\epsilon\rangle \cdot \bar{x}\langle\epsilon\rangle \cdot \text {.nil }\right) \\
& p r_{2} \triangleq ! x() \cdot \overline{a c t}\langle\epsilon\rangle \cdot \overline{x p}\langle\epsilon\rangle \cdot \text { nil } \mid \overline{a c t}\langle\epsilon\rangle \cdot \overline{x p}\langle\epsilon\rangle \text {.nil } \\
& \text { Adeact }_{1} \triangleq \beta^{h}\left(o, r, \Delta^{o}\right) \beta\left(\text { act }, r, \Delta^{+}\right) \beta\left(\text { deact }, r, \Delta^{-}\right) \\
& {\left[p r_{1} \mid ! \bar{o}\langle\epsilon\rangle\right]} \\
& \text { Aact }_{1} \triangleq \beta\left(o, r, \Delta^{o}\right) \beta\left(\text { act }, r, \Delta^{+}\right) \beta\left(\text { deact }, r, \Delta^{-}\right) \\
& {\left[! x() \cdot p s_{1} \mid \overline{\operatorname{deact}}\langle\epsilon\rangle .(\operatorname{hide}(o), \infty) \cdot \bar{x}\langle\epsilon\rangle . \text { nil } \mid ! \bar{o}\langle\epsilon\rangle\right]} \\
& \text { Adeact }_{2} \triangleq \beta^{h}\left(o, r, \Delta^{o}\right) \beta\left(\text { act }, r, \Delta^{+}\right) \beta\left(\text { deact }, r, \Delta^{-}\right) \\
& {\left[p s_{2}\left|p r_{2}\right| ! \bar{o}\langle\epsilon\rangle\right]} \\
& \text { Aact }_{2} \triangleq \beta\left(o, r, \Delta^{o}\right) \beta\left(\text { act }, r, \Delta^{+}\right) \beta\left(\text { deact }, r, \Delta^{-}\right) \\
& {\left[! x() \cdot \overline{a c t}\langle\epsilon\rangle \cdot \overline{x p}\langle\epsilon\rangle \cdot \text { nil }\left|p s s_{2}\right| p s_{2} \mid ! \bar{o}\langle\epsilon\rangle\right]}
\end{aligned}
$$

where $\rho(x)=\rho(x p)=\infty$. The boxes Adeact $_{1}$ and Aact $_{1}$, which uses the pi-processes $p s_{1}$ and $p r_{1}$, represent respectively the deactive and active state of a protein which can be activated by a single external signal. In particular, if the box Adeact $_{1}$ executes an inter-boxes communication through the elementary beta binder $\beta\left(\right.$ act $\left., r, \Delta^{+}\right)$, the action $\overline{a c t}\langle\epsilon\rangle$ in Adeact $_{1}$ is consumed and immediately also the action (unhide $(o), \infty$ ) is consumed (because its rate is $\infty$ ). The obtained box is structurally congruent to Aact 1 and hence the protein has reached its active form, where the elementary beta binder $\beta\left(o, r, \Delta^{o}\right)$ is now unhide and the box can execute inter-communications through it. Now, if the box Aact 1 executes an inter-boxes communication through the elementary beta binder $\beta\left(\right.$ deact $\left., r, \Delta^{-}\right)$, the reverse mechanism is executed and the protein returns back in its deactive form Adeact $_{1}$.

The boxes Adeact $_{2}$ and $A a c t_{2}$, which uses the pi-processes $p s_{2}, p s s_{2}$ and $p r_{2}$, represent respectively the deactive and active state of a protein which can be activated by receiving a signal twice. The activation and deactivation mechanism is similar to the one described for single signal activation.

Obviously these patterns can be easily extended for modelling proteins with more than one sensing and effecting domains and for modeling mechanisms of activation based on the reception of more than two external signals. 


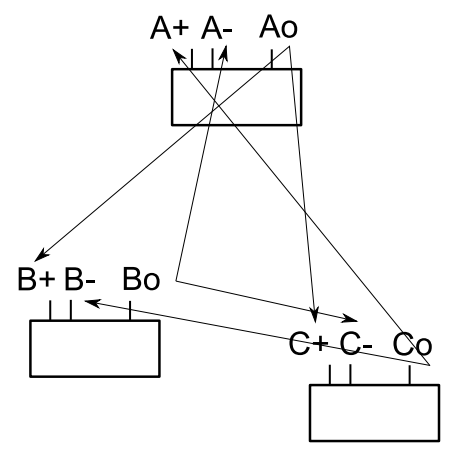

$$
\begin{aligned}
& \boldsymbol{\alpha}_{\mathrm{i}}(\mathrm{Ao}, \mathrm{B}+)=1.0 \\
& \boldsymbol{\alpha}_{\mathrm{i}}(\mathrm{Ao}, \mathrm{C}+)=1.0 \\
& \boldsymbol{\alpha}_{\mathrm{i}}(\text { Bo, A- })=1.0 \\
& \boldsymbol{\alpha}_{\mathrm{i}}(\text { Bo, C- })=1.0
\end{aligned}
$$

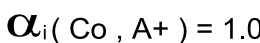

$$
\begin{aligned}
& \boldsymbol{\alpha}_{\mathrm{i}}(\text { Co, B- })=1.0
\end{aligned}
$$

Figure 2: Our representation of protein interaction through sensing and effecting domains. For simplicity we omit subjects and rates of beta binders. Moreover, arrows indicate compatibility of the types, according to the $\alpha$ function specification reported on the right of the figure.

\section{Evolutionary framework}

We design a framework for simulating the evolution of pathways in silico. Evolution proceeds through selection acting on the variance generated by random mutation events. Individuals replicate in proportion to their performance, referred to as fitness. This process can be modelled as shown in Tab. 1. This algorithm differs slightly from the generic evolutionary

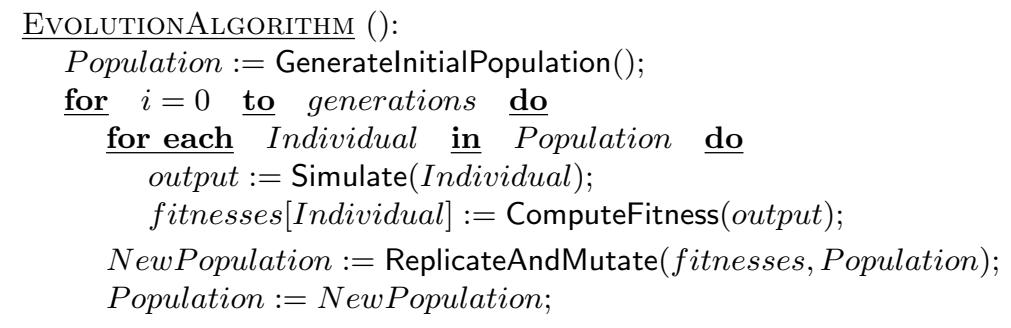

Table 1: Generic EvolutionAlgorithm.

algorithms used in computer science, being more closer to real biological observations made for the asexual reproduction of organisms.

There are four main procedures in the algorithm:

- GenerateInitialPopulation: the initial population can be generated randomly, from a predefined pathway configuration to be used as a starting point, or it can be a pathway with no interactions. All the individuals in the initial population can be equal at the beginning, as they will be differentiated later by the mutation phase.

- Simulate: each individual in the population is simulated separately using the BetaWB stochastic simulator, introduced in Sec. 2.1. 
- ComputeFitness: the output of the simulation is used to compute the fitness value of the current individual. Note that the fitness value is problem-dependent; for an example, refer to Sec. 4 .

- ReplicateAndMutate: this is the most important part of the algorithm; like in a real environment, individuals with the highest fitness values are more likely to survive, replicate and produce a progeny that resembles them, being not, however, completely equal to them.

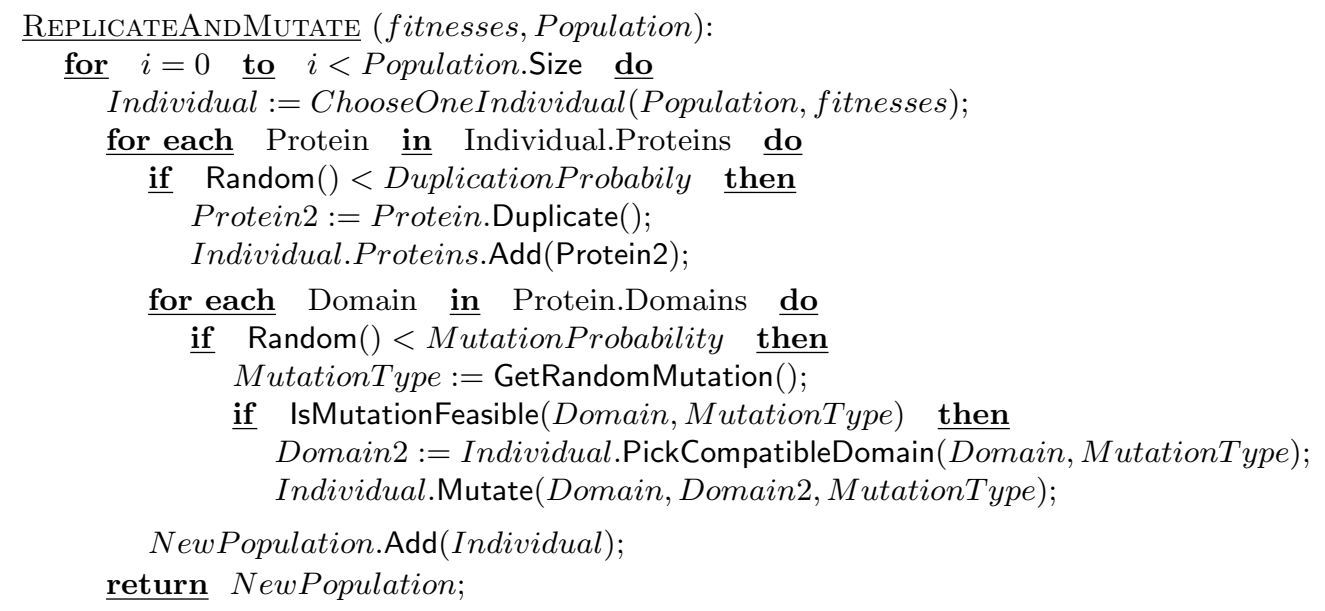

Table 2: The RePlicAteAndMutate algorithm.

The ReplicateAndMutate algorithm (Tab. 2) creates a new population with the same number of individuals of the current generation, using as a base the current individuals. At each step it chooses one individual, with probability proportional to its fitness (CHOOSEONEINDIVIDUAL in the code above). This is achieved by constructing a cumulative probability array $a$ from the fitness array, generating a random number in the range 0...a[Population.Size $]$, and then finding the index into which the random number falls.

The selected individual will replicate and pass to the next generation. During the replication, each protein in the "genome" of the individual is given the chance to mutate, according to a probability.

A mutation is selected among all the possible types by the GETRANDOMMutation function, and this mutation is applied. Finally the individual, that can be equal to its predecessor or can be mutated, is added to the new population. We now define in more detail the kind of mutations that we consider in our framework. 


\subsection{Mutations}

The different types of mutations we consider are based on real biological processes where mutations can happen at DNA and protein level. These ultimately effect pathway dynamics. For example, point mutations in a DNA sequence can change the protein amino-acid sequence, leading to changes in its tertiary structure with implications on the affinity of this protein with other proteins or substrates. Similarly, events at DNA level as gene duplication or domain shuffling can alter pathway structure and dynamics.

A computer program that wants to mimic evolution of a specie have to implement random mutations in individual during replication as well. Here, we can easily implement these molecular processes using the domain and pathway model we discussed in Sect. 2.2.

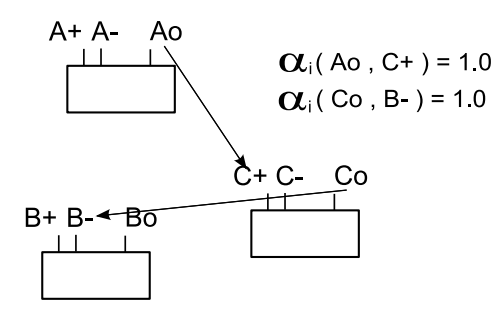

(a)

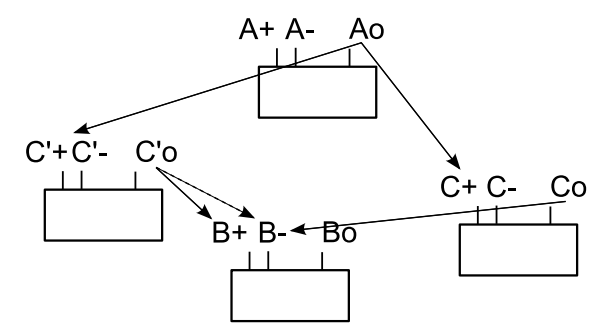

(c)

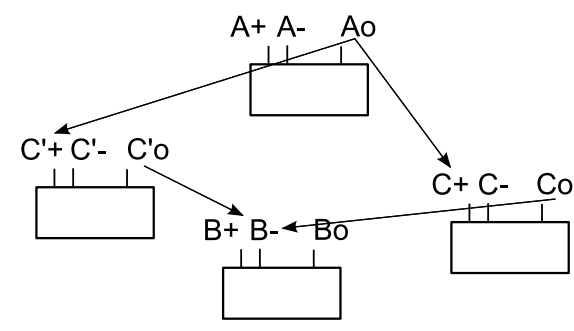

(b)
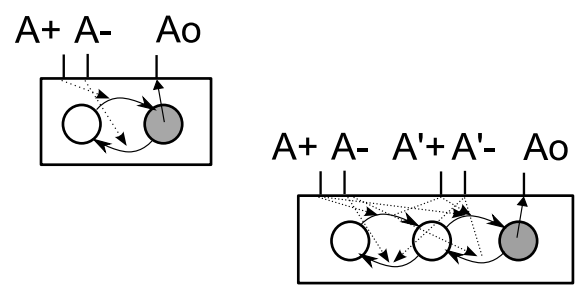

(d)

Figure 3: Different kinds of mutations: in (a) the initial configuration, displaying the $\alpha$ function as a list of tuples; in (b) duplication of protein $C$ followed by mutation of domain $\Delta^{C o}$ in (c). Finally, (d) displays how the internal structure could change to accommodate the duplication of a domain.

We will take as an example the three-protein pathway represented in Fig. 3(a) and we will illustrate how different mutations are possible using the BetaWB language.

\section{Duplication and deletion of proteins:}

the implementation of this mutation consist in duplicating the associated bio-processes. Obviously the name of the bio-processes, as well as the name 
of the types of its binders, must be changed with fresh names. The new types will have the same interaction capabilities, i.e. the same affinities of the original types. Duplication of types is needed because subsequent mutations on one of the binders of the duplicated protein must not affect the original one. Furthermore since the new protein is a new separated entity it must not be structural equivalent to the original one. Following the model presented in Sec. 2.2, the bio-process for protein $C$

$$
\text { Cdeact }_{1} \triangleq \beta^{h}\left(o, r, \Delta^{C o}\right) \beta\left(\text { act }, r, \Delta^{C+}\right) \beta\left(\text { deact }, r, \Delta^{C-}\right)\left[p r_{1} \mid ! \bar{o}\langle\epsilon\rangle\right]
$$

will be duplicated to

$$
\begin{aligned}
& \text { Cdeact }_{1} \triangleq \beta^{h}\left(o, r, \Delta^{C o}\right) \beta\left(\text { act }, r, \Delta^{C+}\right) \beta\left(\text { deact, }, \Delta^{C-}\right)\left[p r_{1} \mid ! \bar{o}\langle\epsilon\rangle\right] \\
& C^{\prime} \text { deact }_{1} \triangleq \beta^{h}\left(o, r, \Delta^{C^{\prime} o}\right) \beta\left(\text { act }, r, \Delta^{C^{\prime}+}\right) \beta\left(\text { deact, }, \Delta^{C^{\prime}-}\right)\left[p r_{1} \mid ! \bar{o}\langle\epsilon\rangle\right]
\end{aligned}
$$

The same duplication will be done also for the bio-process representing the active forms of the protein. Deletion can be implemented removing the associated bio-process, or setting its cardinality to zero.

\section{Mutation of domains:}

the mutation of a domain changes the interaction capabilities of the protein to which it belongs. In our formalism, this is achieved by changing the $\alpha$ function on the two domains that take part in the interaction. More specifically, the mutation on a domain can be a change of interaction, for which we modify the affinity adding a number sampled from a normal distribution with mean zero and a little variance, an addition of an interaction between two domains $d 1$ and $d 2$, modelled as the addition of an affinity $\alpha(d 1, d 2)=x$, whit $x>0$, and finally a removal of an interaction, between two domains $d 1$ and $d 2$, setting $\alpha(d 1, d 2)=0$. For example, the mutation on domain $o C$ that can be observed in Fig.3(c) is obtained by changing the $\alpha$ function from $\alpha(C o, B-)=1.0$ to $\alpha(C o, B-)=0.0, \alpha(C o, B+)=0.9$

\section{Duplication and deletion of domains:}

the last possible mutation, domain duplication or deletion due to shuffling, is more complex as it requires modification of the internal behaviour in response to stimuli. Duplicating or removing domains can be easily done acting on the binders list and on the affinity function $\alpha$; however, for these domains to act as sensing or effecting domains in cooperation or in antagonism with the existing ones, the internal behaviour of the process must also be changed. We devised several possible modifications of the behaviour when a domain is added, such as require communication on all the sensing domains before activating the protein (double phosphorilation, as in 


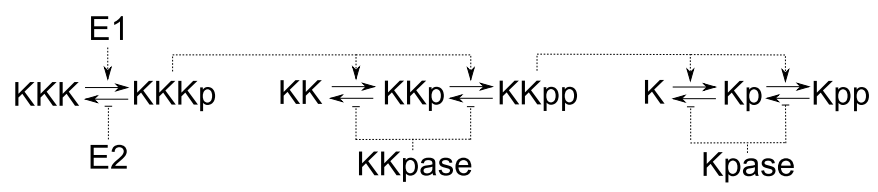

Figure 4: MAPK cascade as described in [16]. $K K K$ denotes $M A P K K K$, $K K$ denotes $M A P K K$ and $K$ denotes $M A P K$. The signal $E 1$ transforms $K K K$ to $K K K p$, which in turn transforms $K K$ to $K K p$ to $K K p p$, which in turn transforms $K$ to $K p$ to $K p p$. In particular, when an input $E 1$ is added, the output of $K p p$ increases rapidly. The transformations in the reverse direction are the result of the signal E2, the KKpase and the Kpase. In particular, by removing the signal $E 1$, the output level of $K p p$ revert back to zero.

Fig. 3(d)), different patterns of activation when more than an effecting domain exists, and so on. All these modifications can be done manipulating the abstract syntax tree of the bio-process, duplicating parts of it in a regular way. However, not all of the possible combinations of transformations have a correspondent biological meaning; a more rigorous investigation on these kind of mutations is to be done before including them in our framework and tool.

\subsection{Measure of fitness}

The measure of fitness is problem dependent: it varies with the kind of pathways, with the characteristics a scientist wants to investigate, and so on. This measure can be done in various ways, including stability analysis, integration of the signal, measure of the derivative. We will illustrate in our example how fitness can be computed using integration of a response.

\section{An example: MAPK cascade}

The mitogen-activated protein kinase cascade (MAPK cascade) is a series of three protein kinases, which is responsible for cell response to growth factors. In [16], an model for the MAPK cascade was presented (Fig.4) and analysed using ODEs; the cascade was shown to perform the function of an ultrasensitive switch and the response curves were shown to be steeply sigmoidal. A process calculi based analysis of the MAPK cascade was presented in [17]. For simplicity, in this paper we rely on a simplified version of the model, where all the enzymatic reactions of the form:

$$
E+S \rightleftharpoons_{K_{E S}^{-1}}^{K_{E S}} E S \rightarrow^{K_{E P}} E P \rightarrow^{\infty} E+P
$$


are substituted with simplest reactions of the form:

$$
E+S \rightarrow{ }^{K_{E P}} E+P
$$

Using the design patterns presented in 2 , a $\beta$-system for the MAPK cascade has been developed (Appendix A). Following [17], we set all the reaction rates to a nominal value of 1.0 and we initialize the system with two of $E 1$, $E 2, K K$ Pase and KPase, 20 of $K K K$ and 200 of $K K$ and $K$. Simulating the MAPK $\beta$-system with the BetaWB simulator, similar response profiles (modulo timescale) were observed for the output of $K p p$ with respect to the model presented in [16], despite the differences in the simulation parameters; the system still behaves as an ultrasensitive switch (Appendix A).

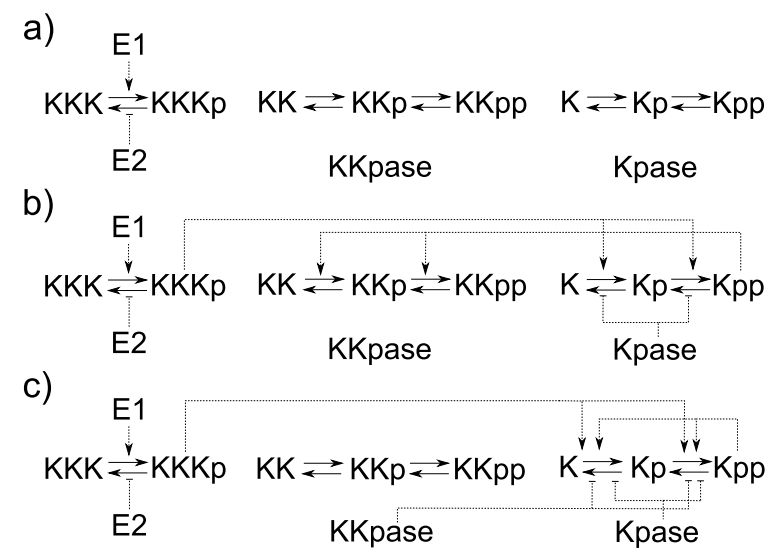

Figure 5: (a) Basic individual of the initial configuration. Only signals $E 1$ and $E 1$ are enabled. (b) A particular individual we obtained, with a "reverse" cascade. (c) A simil-mapk we obtained, with a good fitness value.

We use this simplified MAPK cascade $\beta$-system as a toy model for testing our evolutionary framework. In particular, we want to analyse the evolution of a population according to a fitness function which captures the essential behaviour of our MAPK cascade model.

In detail, we generate an initial population of 500 individuals containing the pathway shown in Fig.5a. This pathway resembles the observed MAPK cascade, in that it contains three kinases, two phosphatases and two signalling molecules. However, it lacks any interactions among these. In other words, we consider an ancestral organism that possessed all these proteins but lacked a signalling system similar to the MAPK cascade as observed today. The dynamic of each individual is then simulated; we run each individual for 7000 simulation steps and we remove the signal $E 1$ at the step 1500. Using the output of the simulation, we then measure for each individual the corresponding fitness. The fitness function we implemented measures how rapidly the output of $K p p$ increases, how much the output 


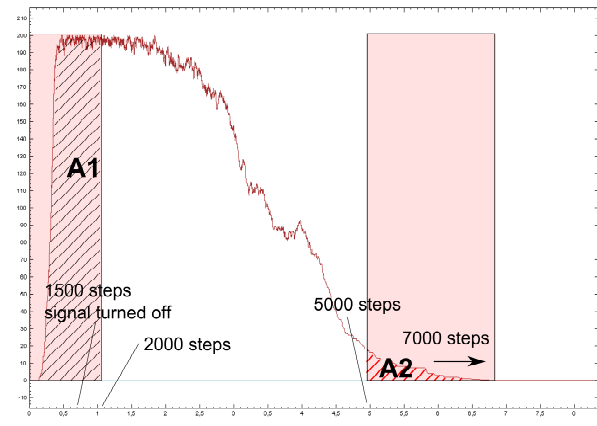

(a)

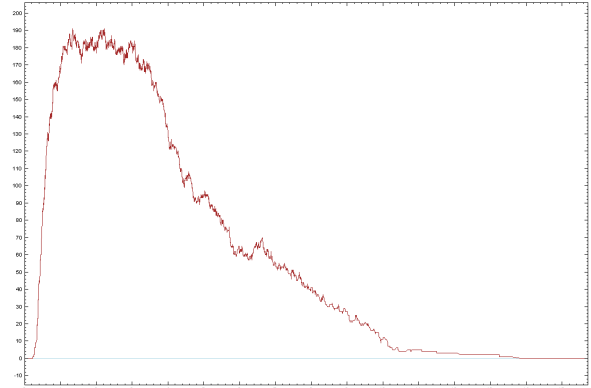

(b)

Figure 6: (a) Time course of the Kpp concentration over the simulation time, superimposed to the integral areas for the fitness function we implemented. The fitness parameters are $i_{1}=0, e_{1}=2000, i_{2}=5000$ and $e_{2}=7000$. (b) An individual with high fitness.

of $K p p$ persists after removing the signal $E 1$ and how rapidly the output of $K p p$ returns back to zero. Let out $=\left\{n_{0}, n_{1}, \ldots, n_{7000}\right\}$ be the tuple representing the Kpp dynamics in time of an individual, then the fitness for out is formally computed by the following formula:

$$
\text { fitness }(\text { out })=\mu+\left(\frac{\sum_{j=i_{1}}^{e_{1}} n_{j}}{K p p_{M} *(e 1-i 1)}-\left(\gamma * \frac{\sum_{j=i_{2}}^{e_{2}} n_{j}}{K p p_{M} *(e 2-i 2)}\right)\right)
$$

The two sums, that we denote respectively with $A 1$ and $A 2$, represent discrete integrals and are normalized with respect to their possible maximum values (see Fig.6). The values $i_{1}, e_{1}, i_{2}$ and $e_{2}$ are changeable parameters that define the boundaries for the computation of the two discrete integrals present in the formula, and the value $K p p_{M}$ represents the maximum value for the $K p p$ respose. Moreover, $\mu$ represents the minimum fitness and $\gamma$ controls the relative importance to responding to a signal and turning the response off after its removal. The reported results are for $i_{1}=0, e_{1}=2000$, $i_{2}=5000, e_{2}=7000, K p p_{M}=200, \mu=0.1$ and $\gamma=0.65$.

According to the algorithms presented in the previous section, the population is evolved. In this case study only mutations of domains are applied. Moreover, in order to maintain a biological validity for the new individuals, possible mutations are the one that satisfies the following constraints: (1) Signals $E 1$ and $E 2$ act only on $K K K$; (2) signal $E 1$ and $E 2$ cannot be removed; (3) a kinase can only activate other kinases or itself; (4) phosphatases are not specific but can only deactivate kinases. We iterate the evolution algorithm for 1000 generations, for different values of fitness function parameters. The dynamic for one of the obtained pathways is shown in Fig.6(b). Examples of obtained individuals are in Fig. 5, while the variation of fitness during a simulation is depicted in Fig. 7. In particular, we do not 

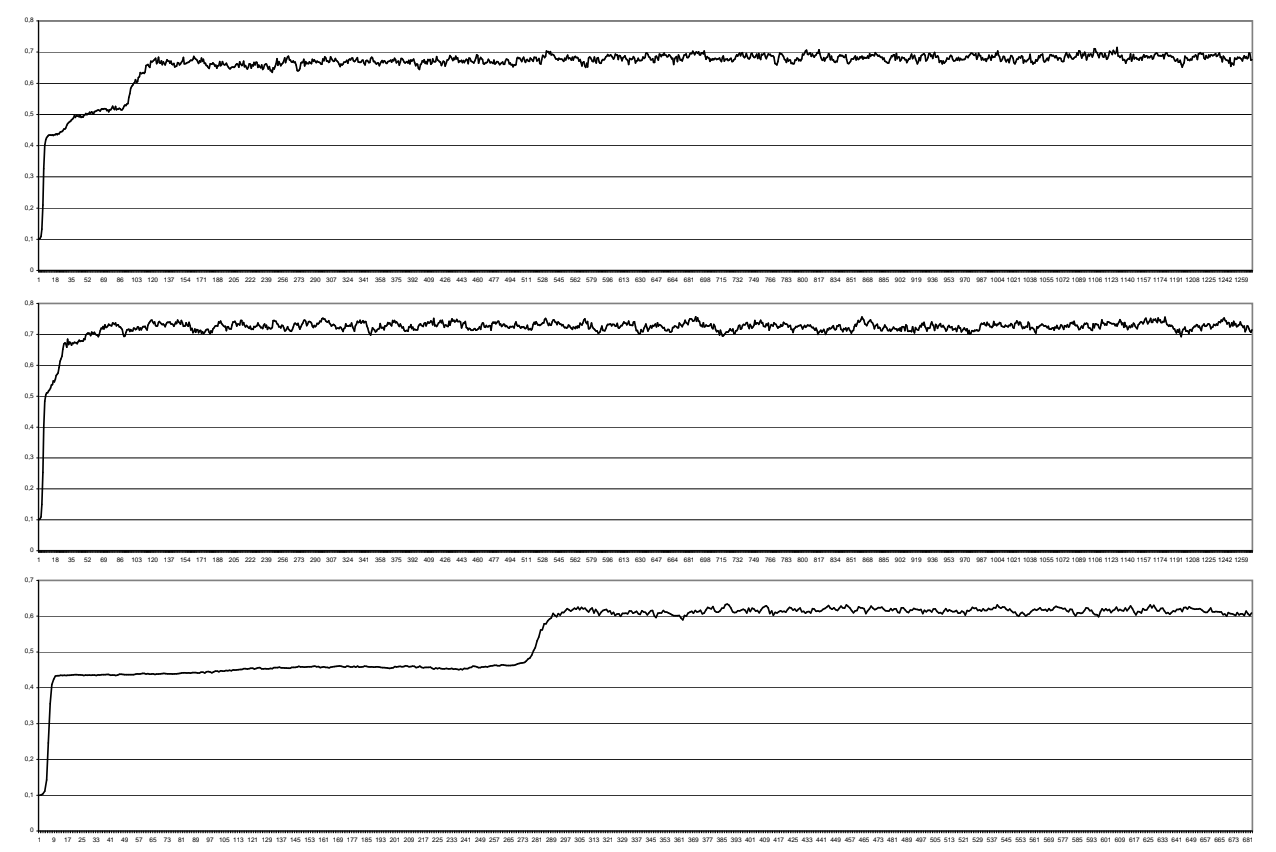

Figure 7: The modification of fitness during three different runs of the in silico evolution.

obtain individuals with a perfect MAPK cascade pathway, but individuals in (b) and (c) are only a reordering and a protein duplication event away from it.

Moreover, we observe three different plateaus on the average fitness variation shown by the first chart in Fig. 7. The first one is obtained after pathways evolve a kinase interaction, the second one is obtained after pathways evolve addition of a phosphatase interaction, and the third one, which persists till the final generation, is obtained after addition of other two interactions -one from a kinase and one from a phosphatase-.

\section{Conclusions and Future work}

In this paper we present a formal approach for simulating the evolution of pathways in silico. Pathway dynamics are described with the BetaWB language, a process algebra that, thanks to its biological-inspired nature, allows for modelling proteins, domains and interactions in a modular way.

We developed a modular description of signalling pathways, an evolutionary algorithm and a prototype to test our concepts. The prototype showed the potential of our approach, and the beta-binders language proved to be well-suited for this task. As presented in the small example in Sec. 4, by simulating evolution we can gain interesting insights in pathway structure 
and on the role of different processes.

We implemented the simpler kinds of mutations, but other interesting variations like modification of internal process structure are feasible and it will surely be a subject worth of future investigation and development.

Also, an easy to use and integrated tool for the simulation and analysis of pathway evolution under development. Finally, we plan to use the same framework also from an optimization perspective to help discovering new pathways.

\section{References}

[1] Regev, A., Shapiro, E.: Cells as computation. Nature (2002)

[2] Priami, C., Regev, A., Shapiro, E., Silvermann, W.: Application of a stochastic name-passing calculus to representation and simulation of molecular processes. Inf. Process. Lett. 80(1) (2001) 25-31

[3] Phillips, A., Cardelli, L.: A correct abstract machine for the stochastic pi-calculus. In: Bioconcur'04, ENTCS (August 2004)

[4] Regev, A., Silverman, W., Shapiro, E.: Representation and simulation of biochemical processes using the pi-calculus process algebra. Pac Symp Biocomput (2001) 459-470

[5] Romanel, A., Dematté, L., Priami, C.: The beta workbench. Technical Report TR03-2007, The Microsoft Research - University of Trento Centre for Computational and Systems Biology (2007)

[6] Sharan, R., Suthram, S., Kelley, R.M., Kuhn, T., McCuine, S., Uetz, P., Sittler, T., Karp, R.M., Ideker, T.: Conserved patterns of protein interaction in multiple species. Proc Natl Acad Sci 6(102) (2005) 1974-79

[7] Babu, M., Teichmann, S., Aravind, L.: Evolutionary dynamics of prokaryotic transcriptional regulators. J. Mol. Biol. (358) (2006) 614-633

[8] Soyer, O., Bonhoeffer, S.: Evolution of complexity in signaling pathways. PNAS (103) (2006) 16337-16342

[9] Pfeiffer, T., Schuster, S.: Game-theoretical approaches to studying the evolution of biochemical systems. Trends Biochem Sci. 1(30) (Jan 2005) 20-5

[10] Pfeiffer, T., Soyer, O., Bonhoeffer, S.: The evolution of connectivity in metabolic networks. PLoS Biol. 7(3) (2005)

[11] P, P.F., Hakim, V.: Design of genetic networks with specified functions by evolution in silico. Proc. Natl. Acad. Sci. 2(101) (2004) 580-5

[12] Fraser, A.S.: Simulation of genetic systems by automatic digital computers. Australian Journal of Biological Science 10 (1957) 484-499

[13] Priami, C., Quaglia, P.: Beta binders for biological interactions. In: CMSB. (2004) $20-33$

[14] Gillespie, D.: Exact stochastic simulation of coupled chemical reactions. J. Phys. Chem. 81(25) (1977) 2340-2361

[15] Stock, A.M., Robinson, V.L., Goudreau, P.N.: Two-component signal transduction. Annu. Rev. Biochem (69) (2000) 183-215

[16] Huang, C.Y., Ferrell, J.E.: Ultrasensitivity in the mitogen-activated protein kinase cascade. Proc Natl Acad Sci U S A 93(19) (September 1996) 10078-10083

[17] Phillips, A., Cardelli, L., Castagna, G. Number 4230 in LNCS. In: A Graphical Representation for Biological Processes in the Stochastic pi-Calculus. Springer (2006) 


\section{AppendixA}

The formal definition of the $\beta$-system of the MAPK cascade is $Z_{M}=$ $\langle B, E, \xi\rangle$ where,

$$
\begin{aligned}
& B=\overbrace{K K K\|\cdots\| K K K}^{20}\|\overbrace{K K\|\cdots\| K K}^{200}\| \overbrace{K\|\cdots\| K}^{200} \| \\
& E_{1}\left\|E_{1}\right\| E_{2}\left\|E_{2}\right\| \text { Kpase \|Kpase \|KKpase \|KKpase } \\
& E=(\text { step }=1500) \operatorname{delete}\left(2, E_{1}\right):: \\
& \xi=(T, \alpha, \rho, \emptyset)
\end{aligned}
$$

The list $E$ contains the enabled events ${ }^{3}$ and the boxes composing the bioprocess $B$ are defined in the following way where we recall that we are using names of pi-processes only to simplify the writing via macro-expansion:

$$
\begin{aligned}
& p s_{1} \triangleq \overline{a c t}\langle\epsilon\rangle \cdot(\text { unhide }(o), \infty) \cdot \overline{\operatorname{deact}}\langle\epsilon\rangle \cdot(\operatorname{hide}(o), \infty) \cdot \bar{x}\langle\epsilon\rangle \cdot \text {.nil } \\
& p r_{1} \triangleq ! x() \cdot p S_{1} \mid p S_{1} \\
& p s s_{2} \triangleq \overline{\operatorname{deact}}\langle\epsilon\rangle \cdot(\operatorname{hide}(o), \infty) \cdot \overline{x p}\langle\epsilon\rangle \cdot \text { nil } \\
& p s_{2} \triangleq ! x p() \cdot\left(\overline{\operatorname{act}}\langle\epsilon\rangle \cdot(\text { unhide }(o), \infty) \cdot p s s_{2}+\overline{\operatorname{deact}}\langle\epsilon\rangle \cdot \bar{x}\langle\epsilon\rangle \cdot \text { nil }\right) \\
& p r_{2} \triangleq ! x() \cdot \overline{\operatorname{act}}\langle\epsilon\rangle \cdot \overline{x p}\langle\epsilon\rangle \cdot \text { nil } \mid \overline{\operatorname{act}}\langle\epsilon\rangle \cdot \overline{x p}\langle\epsilon\rangle \cdot \text {.nil } \\
& \text { pout } \triangleq=! \overline{\text { out }}\langle\epsilon\rangle \cdot \text { nil }
\end{aligned}
$$

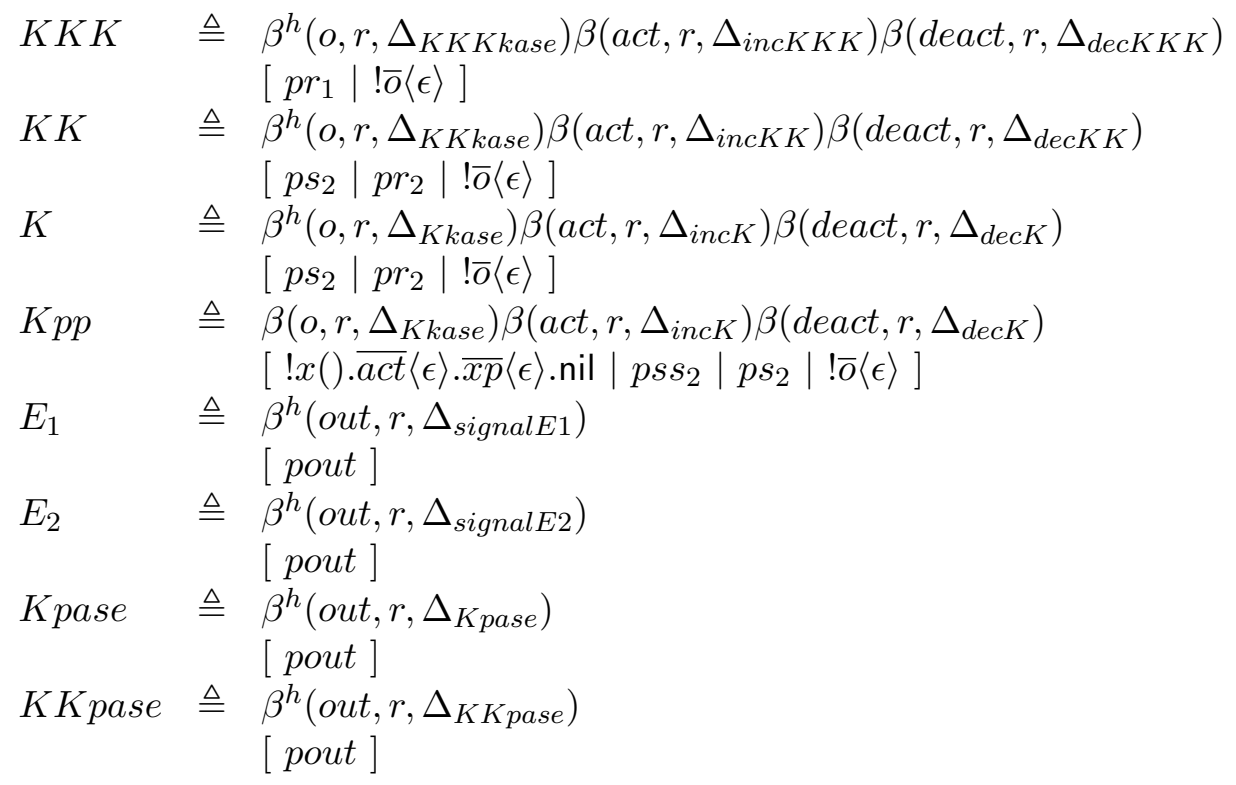

The set of types is

$T=\left\{\Delta_{\text {signalE1 }}, \Delta_{\text {signalE2 } 2}, \Delta_{K K \text { Kkase }}, \Delta_{\text {irecKKK }}\right.$,

\footnotetext{
${ }^{3}$ Timed events, like $($ step $=1500)$ delete $\left(2, E_{1}\right)$, have been implemented although not yet present in the actual definition of the language.
} 
$\Delta_{\text {drecKKK }}, \Delta_{\text {KKkase }}, \Delta_{\text {incKK }}, \Delta_{\text {decKK }}$

$\Delta_{\text {KKpase }}, \Delta_{\text {Kkase }}, \Delta_{\text {incK }}, \Delta_{\text {decK }}$,

$\left.\Delta_{\text {Kpase }}\right\}$

and the $\alpha$ function is defined in the following way:

$$
\begin{array}{ll}
\alpha_{i}\left(\Delta_{\text {irecKKK }}, \Delta_{\text {signalE } 1}\right)=1.0 & \alpha_{i}\left(\Delta_{\text {KK Kase }}, \Delta_{\text {decKK } K}\right)=1.0 \\
\alpha_{i}\left(\Delta_{\text {drecKKK }}, \Delta_{\text {signalE } 2}\right)=1.0 & \alpha_{i}\left(\Delta_{\text {KKkase }}, \Delta_{\text {incK }}\right)=1.0 \\
\alpha_{i}\left(\Delta_{\text {KKKkase }}, \Delta_{\text {incKK }}\right)=1.0 & \alpha_{i}\left(\Delta_{\text {Kpase }}, \Delta_{\text {decK }}\right)=1.0
\end{array}
$$

and the function $\rho$ is such that $\rho(x)=\rho(x p)=\infty$.

The corrisponding source code for the BetaWB simulator is contained in two files. The program file, that contains the structure of the system:

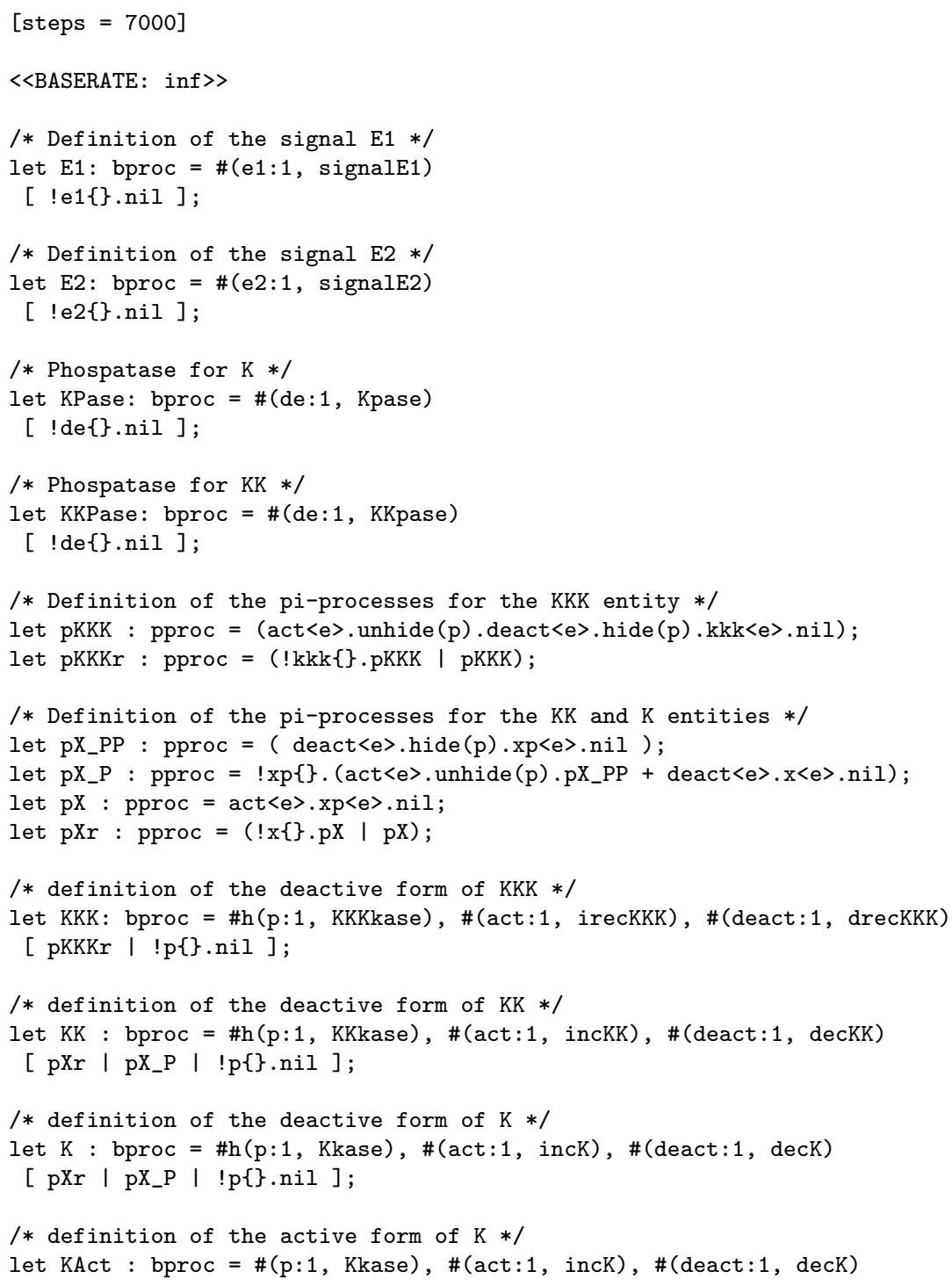




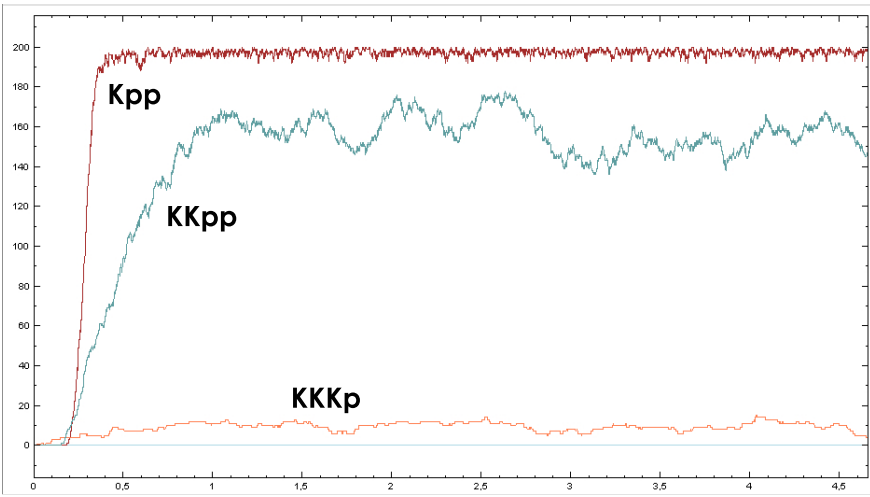

Figure 8: Result of a simulation of the MAPK cascade with the BetaWB simulator.

[ $\left[\mathrm{x}\{\} . \mathrm{pX}\left|\mathrm{pX} \_\mathrm{PP}\right| \mathrm{pX} \_\mathrm{P} \mid \mathrm{p}\{\} . \mathrm{nil}\right]$;

run 2 E1 || 2 E2 || 20 KKK || 200 KK || 2 KKPase || 200 K || 2 KPase

and the type file, that contains the definition of the environment:

\{

signalE1, signale2,

KKKkase, irecKKK, drecKKK,

KKkase, incKK, decKK,

KKpase,

Kkase, incK, decK,

\}

Kpase

$\%$

(irecKKK, signalE1, 1.0),

(drecKKK, signalE2, 1.0),

(KKKkase, incKK, 1.0),

(KKpase, decKK, 1.0),

(KKkase, incK, 1.0),

(Kpase, decK, 1.0)

An example of simulation output, that shows how the system still behaves as an ultrasensitive switch, is in Fig.8. 